\title{
A generalized class of correlated run shock models
}

https://doi.org/10.1515/demo-2018-0008

Received March 9, 2018; accepted May 22, 2018

\begin{abstract}
In this paper, a generalized class of run shock models associated with a bivariate sequence $\left\{\left(X_{i}, Y_{i}\right)\right\}_{i \geq 1}$ of correlated random variables is defined and studied. For a system that is subject to shocks of random magnitudes $X_{1}, X_{2}, \ldots$ over time, let the random variables $Y_{1}, Y_{2}, \ldots$ denote times between arrivals of successive shocks. The lifetime of the system under this class is defined through a compound random variable $T=\sum_{t=1}^{N} Y_{t}$, where $N$ is a stopping time for the sequence $\left\{X_{i}\right\}_{i \geq 1}$ and represents the number of shocks that causes failure of the system. Another random variable of interest is the maximum shock size up to $N$, i.e. $M=\max \left\{X_{i}, 1 \leq i \leq N\right\}$. Distributions of $T$ and $M$ are investigated when $N$ has a phase-type distribution.
\end{abstract}

Keywords: Compound distributions, Dependence, Laplace transform, Phase-type distributions, Shock models

\section{Introduction}

Reliability shock models have always attracted attention in the literature. In a typical shock model, a system (or component) is subject to shocks of random magnitudes at random times and it fails according to a certain rule corresponding to shocks and/or times between shocks. With respect to the failure criteria of the system, shock models can be classified as cumulative shock models, extreme shock models, run shock models, delta shock models, and mixed shock models. Sumita and Shanthikumar [17] defined and studied a class of cumulative shock models. Anderson [1] established limit theorems for the system failure time under the cumulative shock model. Gut [7] used the theory for stopped two-dimensional random walks to describe cumulative shock models. Gut and Hüsler [9] studied extreme shock models. Gut [8] introduced and studied mixed shock models. Cirillo and Hüsler [2] proposed an alternative approach to extreme shock models using reinforced urn processes. Li et al. [10] have studied lifetime properties of the $\delta$-shock model under the classical assumption that the shocks arrive according to a Poisson process. Li and Kong [11] investigated a $\delta$-shock model under nonhomogeneous Poisson process of shock arrivals. Eryilmaz [3] generalized the $\delta$-shock model using the concept of runs. Eryilmaz [4] studied the discrete time version of the $\delta$-shock model in which the shocks occur according to a binomial process, i.e. the interarrival times between successive shocks follow a geometric distribution with mean 1/p. Parvardeh and Balakrishnan [16] discussed a mixed $\delta$-shock model.

If the random variable $N$ denotes the number of shocks that causes failure of the system and $Y_{1}, Y_{2}, \ldots$ represent the times between arrivals of successive shocks, then the system's failure time can be represented as $T=\sum_{t=1}^{N} Y_{t}$. The type of the shock model is defined through the random variable $N$. For example, in the extreme shock model, the random variable $N$ counts the number of shocks until the first shock above

Femin Yalcin: Department of Engineering Sciences, Izmir Katip Celebi University, 35620, Balatcik, Cigli, Izmir, Turkey, E-mail: femin.yalcin@ikc.edu.tr

*Corresponding Author: Serkan Eryilmaz: Department of Industrial Engineering, Atilim University, 06836, Incek, Ankara, Turkey, E-mail: serkan.eryilmaz@atilim.edu.tr

Ali Riza Bozbulut: Department of Industrial Engineering, Atilim University, 06836, Incek, Ankara, Turkey, E-mail:

ali.bozbulut@atilim.edu.tr 
a critical level, i.e., a large shock. For cumulative, extreme, and run shock models, the random variable $N$ depends on the magnitudes $X_{1}, X_{2}, \ldots$ of shocks. Two different cases can be considered to study the shock models. In the first and classical case, the two sequences $\left\{Y_{i}\right\}_{i \geq 1}$ and $\left\{X_{i}\right\}_{i \geq 1}$ are assumed to be independent while in the second case there is a correlation between $X_{i}$ and $Y_{i}$ for all $i$. The assumption of independence between the sequences $\left\{Y_{i}\right\}_{i \geq 1}$ and $\left\{X_{i}\right\}_{i \geq 1}$ can be restrictive in practical contexts. For example, $X_{1}, X_{2}, \ldots$ may represent intensities of successive catastrophes. In modelling natural catastrophic events, it is logical to assume a dependence between the intensity of the catastrophe and the time elapsed since the previous catastrophe [14]. Ozkut and Bayramoglu (Bairamov) [15] studied Marshall-Olkin type shock model with the assumption that if the magnitude of the shock exceeds some predefined threshold, then the component, which is subjected to this shock, is destroyed; otherwise it survives. More precisely, they assumed that the shock time and the magnitude of the shock are dependent random variables with a bivariate distribution.

Mallor and Omey [12] studied a run shock model assuming dependence between $X_{i}$ and $Y_{i}$ for all $i$. In their model, the random variable $N$ counts the number of shocks until the first $k$ consecutive critical shocks and it has geometric distribution of order $k$ which has been shown to be a phase-type distribution (for further details see [18]). In particular, Mallor and Omey [12] obtained the distribution of $T=\sum_{t=1}^{N} Y_{t}$ when $N$ has geometric distribution of order $k$. They have also obtained the distribution of the maximum shock size up to $N$, i.e. $M=\max \left\{X_{i}, 1 \leq i \leq N\right\}$ when $N$ has geometric distribution of order $k$. In the present paper, we aim to generalize the model studied by Mallor and Omey [12] by assuming that the random variable $N$ has an arbitrary phase-type distribution. With this generalization, we will be able to define a new class of shock models and obtain matrix-based representations for reliability characteristics associated with the shock model. This new generalization also extends the results in [5] where a certain class of shock models for the case when the times between arrivals of shocks and the magnitudes of shocks are independent. In the class defined by Eryilmaz [5], the failure time of the system is represented by $T=\sum_{t=1}^{N} Y_{t}$, and the random variable $N$ which is independent of $Y \mathrm{~s}$ is assumed to have a phase-type distribution. If the random variables $N$ and $Y_{1}, Y_{2}, \ldots$ are independent and have phase-type distributions, then according to the well-known closure property of phase-type random variables, the random sum $\sum_{t=1}^{N} Y_{t}$ has also a phase-type distribution. On the other hand, if there is a dependence between $N$ and $Y$ s, then the random variable $\sum_{t=1}^{N} Y_{t}$ may not have a phase-type distribution.

The paper is organized as follows. In Section 2, we present distributions of $T$ and $M$ when the random variable $N$ has a phase-type distribution. Section 3 contains a generalized run shock model. In Section 4, we present graphical illustrations.

\section{Distributions of $T$ and $M$}

For a system that is subject to shocks of random magnitudes $X_{1}, X_{2}, \ldots$ over time, let $Y_{1}, Y_{2}, \ldots$ denote times between arrival of successive shocks. Let $N$ be a stopping time for the sequence $X_{1}, X_{2}, \ldots$ (i.e. the event $\{N=n\}$ is independent of $X_{n+1}, X_{n+2}, \ldots$ for all $\left.n=1,2, \ldots\right)$, and $\left(X_{1}, Y_{1}\right),\left(X_{2}, Y_{2}\right), \ldots$ be a sequence of independent and identically distributed (iid) random vectors with common continuous cumulative distribution function (cdf) $F(x, y)=P\left\{X_{i} \leq x, Y_{i} \leq y\right\}, i=1,2, \ldots$.

It is assumed that the stopping time random variable $N$ is defined on a binary sequence $I_{1}, I_{2}, \ldots$ of random variables such that $I_{i}=1$ if $X_{i} \in R$, and $I_{i}=0$ if $X_{i} \notin R$, where $R \subset[0, \infty)$ can be considered as a critical region and the event $X_{i} \in R$ can be thought as a critical event (e.g. a large shock). Thus the random variable $N$ is simply a waiting time that counts the number of events until the occurrence of a certain rule by the random variables $I_{1}, I_{2}, \ldots$ (e.g. $N$ may represent the number of shocks until a run of $k$ consecutive critical (large) shocks). Then the compound random variable $T=\sum_{t=1}^{N} Y_{t}$ defines the failure time of the system. In the class of shock models that will be considered throughout the present paper, the failure time of the system will be represented by a compound $\operatorname{sum} \sum_{t=1}^{N} Y_{t}$, where $N$ is assumed to have a phase-type distribution. 
If the random variable $N$ that is defined on the binary sequence $I_{1}, I_{2}, \ldots$ has a phase-type distribution of order $d$, then its probability mass function (pmf) can be written as

$$
P\{N=n ; p, 1-p\}=\pi \mathbf{Q}^{n-1} \mathbf{u}^{\prime},
$$

for $n \in \mathbb{N}$, where $\mathbf{Q}=\left(q_{i j}\right)_{d \times d}$ is a matrix which includes the transition probabilities among the $d$ transient states, $\mathbf{u}^{\prime}=\left(\mathbf{I}_{d}-\mathbf{Q}\right) \mathbf{e}^{\prime}$ is a vector which includes the transition probabilities from transient states to the absorbing state, $\boldsymbol{\pi}=\left(\pi_{1}, \ldots, \pi_{d}\right)$ with $\sum_{i=1}^{d} \pi_{i}=1, \mathbf{I}_{d}$ is the identity matrix with dimension $d \times d$, and $\mathbf{e}=(1, \ldots, 1)_{1 \times d}$ (for further details see [13]). The matrix $\mathbf{Q}$ must satisfy the condition that $\mathbf{I}_{d}-\mathbf{Q}$ is nonsingular. The probability generating function (pgf) of $N$ is given by

$$
\phi_{N}(z)=1-\pi \mathbf{e}^{\prime}+\pi z\left(\mathbf{I}_{d}-z \mathbf{Q}\right)^{-1} \mathbf{u}^{\prime},
$$

for $0<z<1$. The expected value of $N$ can be computed from $E(N)=\pi\left(\mathbf{I}_{d}-\mathbf{Q}\right)^{-1} \mathbf{e}^{\prime}$. The parameter $p$ in (1) represents the probability that the magnitude of a shock falls into the critical region, i.e., $p=P\left(X_{i} \in R\right), i \geq 1$.

\subsection{Distribution of $T$}

Let $\xi_{N}$ denote the number of critical events until the failure of the system. Define

$$
L_{1}(s)=E\left(e^{-s Y} \mid X \notin R\right)
$$

and

$$
L_{2}(s)=E\left(e^{-s Y} \mid X \in R\right) .
$$

In the following, we present an expression for the Laplace-Stieltjes transform (LST) of $T$ for an arbitrary stopping time random variable $N$. The result in Proposition 1 is continuous version of Proposition 1 in [6], and hence its proof is omitted.

Proposition 1 Let $N$ be a stopping time for the sequence $\left\{X_{i}\right\}_{i \geq 1}$ and $\left\{\left(X_{i}, Y_{i}\right)\right\}_{i \geq 1}$ be a sequence of iid random vectors. Then the LST of $T$ is

$$
T^{\star}(s)=E\left(e^{-s T}\right)=E\left(\left(L_{2}(s)\right)^{\xi_{N}}\left(L_{1}(s)\right)^{N-\xi_{N}}\right),
$$

where $\xi_{N}=\sum_{i=1}^{N} I_{i}=\sum_{i=1}^{N} I\left(X_{i} \in R\right)$

If $N$ has a phase-type distribution of order $d$ with pmf (1), then the joint pgf of $\left(N-\xi_{N}, \xi_{N}\right)$ is given by

$$
\phi\left(z_{1}, z_{2}\right)=\boldsymbol{\pi}\left(\mathbf{I}_{d}-\mathbf{Q}_{z_{1}, z_{2}}\right)^{-1} \mathbf{u}_{z_{1}, z_{2}}^{\prime},
$$

where $\mathbf{Q}_{z_{1}, z_{2}}$ and $\mathbf{u}_{z_{1}, z_{2}}^{\prime}$ are obtained replacing $p$ and $1-p$ in $\mathbf{Q}$ and $\mathbf{u}^{\prime}$ respectively by $p z_{2}$ and $(1-p) z_{1}[6]$.

Hence, we can obtain the following expression for the LST of the random variable $T=\sum_{t=1}^{N} Y_{t}$ whenever $N$ has a phase-type distribution. Such a matrix representation for the LST of the lifetime random variable $T$ is new in the literature.

Theorem 1 Let $N$ have a phase-type distribution of order $d$ with pmf

$P\{N=n ; p, 1-p\}=\pi \mathbf{Q}^{n-1} \mathbf{u}^{\prime}$, where $p=P\left(X_{i} \in R\right)$. Then the LST of $T$ is

$$
T^{\star}(s)=E\left(e^{-s T}\right)=\pi\left(\mathbf{I}_{d}-\mathbf{Q}_{s}\right)^{-1} \mathbf{u}_{s}^{\prime},
$$

where $\mathbf{Q}_{s}$ and $\mathbf{u}_{s}^{\prime}$ are obtained replacing $p$ and $1-p$ in $\mathbf{Q}$ and $\mathbf{u}^{\prime}$ respectively by $p L_{2}(s)$ and $(1-p) L_{1}(s)$. Proof The proof immediately follows from Proposition 1 and (4) because $E\left(e^{-s T}\right)=$ $E\left(\left(L_{2}(s)\right)^{\xi_{N}}\left(L_{1}(s)\right)^{N-\xi_{N}}\right)=\phi\left(L_{1}(s), L_{2}(s)\right)$

Example 1 Consider the run shock model defined by Mallor and Omey [12]. In this case, the random variable $N$ counts the number of shocks until $k$ consecutive critical shocks, and it has a phase-type distribution of order $k$ with $\pi=(1,0, \ldots, 0)$,

$$
\mathbf{Q}=\left[\begin{array}{ccccc}
1-p & p & 0 & \cdots & 0 \\
1-p & 0 & p & \cdots & 0 \\
\vdots & \vdots & \vdots & \vdots & \vdots \\
1-p & 0 & 0 & \cdots & 0
\end{array}\right]
$$


and $\mathbf{u}=(0, \ldots, 0, p)$, where $p=P\left(X_{i} \in R\right)$. Using Theorem 1, the LST of lifetime of the system $T=\sum_{t=1}^{N} Y_{t}$ is obtained as

$$
\begin{aligned}
T^{\star}(s)= & (1,0, \ldots, 0)\left[\left[\begin{array}{cccc}
1 & 0 & \cdots & 0 \\
0 & 1 & \cdots & 0 \\
\vdots & \vdots & \vdots & \vdots \\
0 & 0 & \cdots & 1
\end{array}\right]-\left[\begin{array}{ccccc}
(1-p) L_{1}(s) & p L_{2}(s) & 0 & \cdots & 0 \\
(1-p) L_{1}(s) & 0 & p L_{2}(s) & \cdots & 0 \\
\vdots & \vdots & \vdots & \vdots & \vdots \\
(1-p) L_{1}(s) & 0 & 0 & \cdots & 0
\end{array}\right]\right)^{-1} \\
\times & {\left[\begin{array}{c}
0 \\
\vdots \\
0 \\
p L_{2}(s)
\end{array}\right] }
\end{aligned}
$$

where $L_{1}(s)=E\left(e^{-s Y} \mid X \notin R\right)$ and $L_{2}(s)=E\left(e^{-s Y} \mid X \in R\right)$.

Equation (6) is an alternative to the recursive expression given in Theorem 2.1 of Mallor and Omey [12].

As it can be seen from Theorem 1, to obtain the LST of $T$ it is enough to find an expression for $L_{1}(s)$. Let $F(x, y)=P\{X \leq x, Y \leq y\}$ be the joint cdf of $X$ and $Y$, and $F_{X}\left(F_{Y}\right)$ and $\bar{F}_{X}\left(\bar{F}_{Y}\right)$ be the marginal cdfs and survival functions of $X(Y)$, respectively. If the critical region is chosen to be $R=(c, \infty)$, then

$$
\begin{aligned}
L_{1}(s) & =E\left(e^{-s Y} \mid X \notin R\right) \\
& =E\left(e^{-s Y} \mid X \leq c\right) \\
& =\int_{0}^{\infty} e^{-s y} \int_{0}^{c} f_{Y \mid X}(y \mid x) d x d y \\
& =\frac{1}{F_{X}(c)} \int_{0}^{\infty} e^{-s y} \int_{0}^{c} f(x, y) d x d y
\end{aligned}
$$

Since

$$
\begin{aligned}
E\left(e^{-s Y}\right) & =E\left(e^{-s Y} \mid X \notin R\right) P\{X \notin R\}+E\left(e^{-s Y} \mid X \in R\right) P\{X \in R\} \\
& =E\left(e^{-s Y} \mid X \leq c\right) P\{X \leq c\}+E\left(e^{-s Y} \mid X>c\right) P\{X>c\} \\
& =L_{1}(s) F_{X}(c)+L_{2}(s) \bar{F}_{X}(c),
\end{aligned}
$$

we have

$$
L_{2}(s)=\frac{1}{\bar{F}_{X}(c)} \int_{0}^{\infty} e^{-s y} \int_{c}^{\infty} f(x, y) d x d y .
$$

Example 2 Assume that the joint cdf of $X$ and $Y$ is modeled by the Farlie-Gumbel-Morgenstern (FGM) distribution which is given by

$$
F(x, y)=F_{X}(x) F_{Y}(y)\left\{1+\alpha\left(1-F_{X}(x)\right)\left(1-F_{Y}(y)\right)\right\}
$$

$-1 \leq \alpha \leq 1$. Then the conditional density of $Y$ given $X \leq c$ is

$$
f_{Y \mid X \leq c}(y)=f_{Y}(y)\left[1+\alpha \bar{F}_{X}(c)\left(1-2 F_{Y}(y)\right)\right] .
$$

Suppose that $F_{X}(x)=1-e^{-\lambda x}, x \geq 0$, and $F_{Y}(y)=1-e^{-\theta y}, y \geq 0$. Then

$$
L_{1}(s)=\frac{\theta\left(1-\alpha e^{-\lambda c}\right)}{s+\theta}+\frac{2 \alpha \theta e^{-\lambda c}}{s+2 \theta}
$$

and

$$
L_{2}(s)=\frac{\theta\left(1+\alpha\left(1-e^{-\lambda c}\right)\right)}{s+\theta}-\frac{2 \alpha \theta\left(1-e^{-\lambda c}\right)}{s+2 \theta} .
$$




\subsection{Distribution of $M$}

Let

$$
P(x)=P\{X \leq X, X \in R\},
$$

and

$$
Q(x)=P\{X \leq x, X \notin R\} .
$$

The following theorem gives the cumulative distribution function of $M=\max \left\{X_{i}\right.$,

$1 \leq i \leq N\}$ for an arbitrary phase-type random variable having pmf (1). It extends Lemma 2.2 of Mallor and Omey [12].

Theorem 2 Let $N$ have a phase-type distribution of order $d$ with pmf $P\{N=n ; p, 1-p\}=\pi \mathbf{Q}^{n-1} \mathbf{u}^{\prime}$, where $p=P\left(X_{i} \in R\right)$. Then the cdf of $M$ is

$$
P\{M \leq x\}=\pi\left(\mathbf{I}_{\mathbf{d}}-\mathbf{Q}_{x}\right)^{-1} \mathbf{u}_{x}^{\prime},
$$

where $\mathbf{Q}_{x}$ and $\mathbf{u}_{x}^{\prime}$ are obtained replacing $p$ and $1-p$ in $\mathbf{Q}$ and $\mathbf{u}^{\prime}$ respectively by $P(x)$ and $Q(x)$.

Proof By conditioning on $N$ and $\xi_{N}$,

$$
\begin{aligned}
P\{M \leq x\} & =\sum_{n} \sum_{a} P\left\{X_{1} \leq x, \ldots, X_{n} \leq x, N=n, \xi_{n}=a\right\} \\
& =\sum_{n} \sum_{a} C_{n}(a)[P\{X \leq x, X \in R\}]^{a}[P\{X \leq x, X \notin R\}]^{n-a},
\end{aligned}
$$

where $C_{n}(a)$ is the number of binary sequences $I_{1}, \ldots, I_{n}$ satisfying $\left\{N=n, \xi_{n}=a\right\}$. The sum

$$
\sum_{a} C_{n}(a)[P\{X \leq x, X \in R\}]^{a}[P\{X \leq X, X \notin R\}]^{n-a}
$$

is in fact the pmf of $N$ when $p$ and $1-p$ are replaced respectively by $P(x)$ and $Q(x)$. Thus

$$
\begin{aligned}
P\{M \leq x\} & =\sum_{n} P\{N=n ; P(x), Q(x)\} \\
& =\sum_{n} \pi \mathbf{Q}_{x}^{n-1} \mathbf{u}_{x}^{\prime} \\
& =\pi\left(\mathbf{I}_{\mathbf{d}}-\mathbf{Q}_{x}\right)^{-1} \mathbf{u}_{x}^{\prime}
\end{aligned}
$$

which completes the proof.

\section{Generalized run shock model}

Consider the situation when the system fails due to the occurrence of $m$ nonoverlapping runs of $k$ consecutive critical shocks. Assume that the values of the random variables $I_{1}, I_{2}, \ldots$ are 010001101001011 . For $k=2$, if $m=1$ then the number of shocks until the failure of the system is 7 and if $m=2$, then the number of shocks until the failure of the system is 15 . Such a model is useful if there are $m$ cold standby identical systems since in this case the system needs to be replaced with a new one after the occurrence of $k$ consecutive critical shocks. If $N_{m}(k)$ denotes the number of shocks until $m$ nonoverlapping runs of $k$ consecutive critical shocks, then the system's lifetime can be expressed as

$$
T=\sum_{t=1}^{N_{m}(k)} Y_{t} .
$$

The random variable $N_{m}(k)$ is in fact the sum of $m$ independent phase-type random variables each having geometric distribution of order $k$. By the closure property of phase-type distributions, $N_{m}(k)$ has also a phasetype distribution. The pmf of $N_{m}(k)$ can be written as

$$
P\left\{N_{m}(k)=n ; p, 1-p\right\}=\pi \mathbf{Q}_{m}^{n-1} \mathbf{u}_{m}^{\prime},
$$


where $\pi$ is a $1 \times m k$ vector given by $\pi=(1,0, \ldots, 0)$,

$$
\mathbf{Q}_{m}=\left[\begin{array}{cc}
\mathbf{Q}_{m-1} & \mathbf{A} \\
\mathbf{0} & \mathbf{B}
\end{array}\right],
$$

for $m \geq 3$, and $\mathbf{u}_{m}^{\prime}=\left(\mathbf{I}_{m k}-\mathbf{Q}_{m}\right) \mathbf{e}^{\prime}[18] . \mathbf{Q}_{m}$ is an $m k \times m k$ matrix and

$$
\mathbf{Q}_{2}=\left[\begin{array}{ccccccccc}
1-p & p & 0 & \cdots & 0 & 0 & 0 & \cdots & 0 \\
1-p & 0 & p & \cdots & 0 & 0 & 0 & \cdots & 0 \\
\vdots & \vdots & \vdots & \vdots & \vdots & \vdots & \vdots & \vdots & \vdots \\
1-p & 0 & 0 & \cdots & 0 & p & 0 & \cdots & 0 \\
0 & 0 & 0 & \cdots & 0 & 1-p & p & \cdots & 0 \\
0 & 0 & 0 & \cdots & 0 & 1-p & 0 & \cdots & 0 \\
\vdots & \vdots & \vdots & \vdots & \vdots & \vdots & \vdots & \vdots & \vdots \\
0 & 0 & 0 & \cdots & 0 & 1-p & 0 & \cdots & 0
\end{array}\right]_{2 k \times 2 k}
$$

The matrices $\mathbf{A}$ and $\mathbf{B}$ with respective dimensions $(m-1) k \times k$ and $k \times k$ are given by

$$
\mathbf{A}=\left[\begin{array}{ccccc}
0 & 0 & 0 & \cdots & 0 \\
\vdots & \vdots & \vdots & \cdots & \vdots \\
0 & 0 & 0 & \vdots & 0 \\
p & 0 & 0 & \cdots & 0
\end{array}\right] \text { and } \mathbf{B}=\left[\begin{array}{ccccc}
1-p & p & 0 & \cdots & 0 \\
1-p & 0 & p & \cdots & 0 \\
\vdots & \vdots & \vdots & \vdots & \vdots \\
1-p & 0 & 0 & \cdots & 0
\end{array}\right]
$$

Thus the LST of $T$ can be computed replacing $p$ and $1-p$ in $\mathbf{Q}_{m}$ and $\mathbf{u}_{m}^{\prime}$ respectively by $p L_{2}(s)$ and $(1-p) L_{1}(s)$ and using (5).

Example 3 Let $m=3$ and $k=2$. Then

$$
\begin{gathered}
P\left\{N_{m}(k)=n ; p, 1-p\right\} \\
=(1,0,0,0,0,0)\left[\begin{array}{cccccc}
1-p & p & 0 & 0 & 0 & 0 \\
1-p & 0 & p & 0 & 0 & 0 \\
0 & 0 & 1-p & p & 0 & 0 \\
0 & 0 & 1-p & 0 & p & 0 \\
0 & 0 & 0 & 0 & 1-p & p \\
0 & 0 & 0 & 0 & 1-p & 0
\end{array}\right]^{n-1}\left[\begin{array}{l}
0 \\
0 \\
0 \\
0 \\
0 \\
p
\end{array}\right],
\end{gathered}
$$

for $n=6,7, \ldots$. Using Theorem 1, the LST of the system's lifetime is obtained as

$$
T^{\star}(s)=\frac{\left(p L_{2}(s)\right)^{6}}{\left[1-(1-p) L_{1}(s)\left(1+p L_{2}(s)\right)\right]^{3}} .
$$

Using Theorem 2, the cdf of $M$ is found to be

$$
P\{M \leq x\}=\frac{P^{6}(x)}{[1-P(x)(1+Q(x))]^{3}} .
$$

\section{Graphical illustrations}

In this section, we present some numerical results to compare the distribution of the system failure time for the two cases when $X_{i}$ and $Y_{i}$ are independent (Case 1) and when $X_{i}$ and $Y_{i}$ are dependent (Case 2). For the dependent case, the joint distribution of $X$ and $Y$ is assumed to be FGM which is given in (7). For both cases, 
the marginals are chosen to be exponential with $F_{X}(x)=1-e^{-\lambda x}, x \geq 0$, and $F_{Y}(y)=1-e^{-\theta y}, y \geq 0$. In Figures $1-2$, we plot survival functions of the system under the generalized run shock model defined in Section 3 when $\lambda=1, \theta=0.5$ for Case 1(the dashed line) and when $\lambda=1, \theta=0.5, \alpha=0.95$ for Case 2 (the solid line). In both cases, the probability of critical event is $p=P\left(X_{i}>c\right)=0.2$. As it is clear from the figures, one survival function is not larger than the other for all values of $t$.

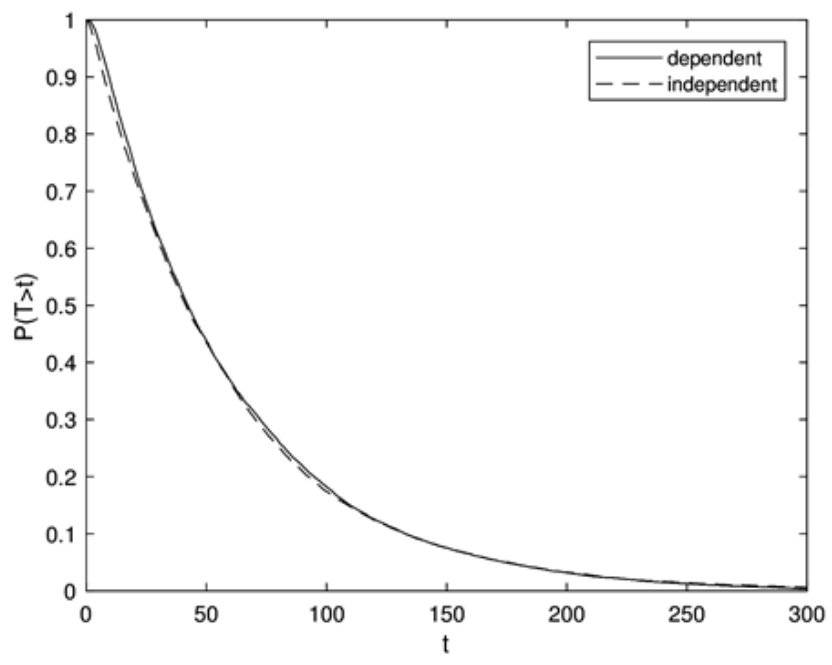

Figure 1: Survival functions for Case 1 and Case 2 when $m=1$ and $k=2$.

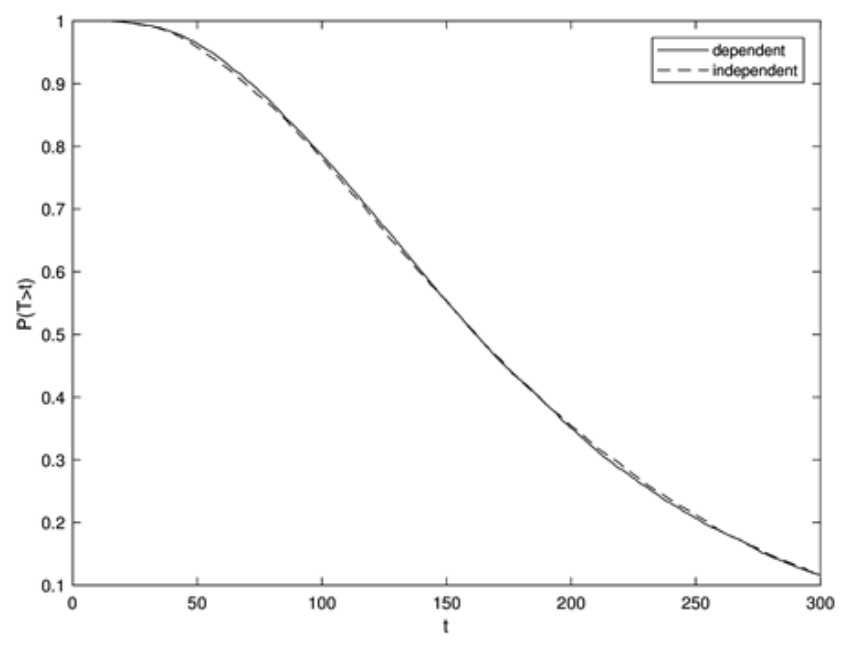

Figure 2: Survival functions for Case 1 and Case 2 when $m=3$ and $k=2$. 


\section{Summary and Conclusions}

In this paper, we have introduced a class of run shock models associated with a bivariate sequence $\left\{\left(X_{i}, Y_{i}\right)\right\}_{i \geq 1}$ of correlated random variables. In this class, the number of shocks that causes failure of the system is assumed to have a phase-type distribution. We have presented matrix-based analytical results for the distribution of the system's lifetime and the distribution of the maximum shock size at the time when the system fails. We have defined a new generalized run shock model in which the system fails due to the occurrence of $m$ nonoverlapping runs of $k$ consecutive critical shocks. The model defined by Mallor and Omey [12] is a special case of this model when $m=1$. The class and the corresponding results might be useful not only in the context of reliability but also in insurance. In insurance, the random variables $X$ and $Y$ respectively represent claim size and interclaim time.

Acknowledgement: The authors thank two reviewers for their useful comments and suggestions.

\section{References}

[1] Anderson, K. K. (1988). A note on cumulative shock models. J. Appl. Probab. 25(1), 220-223.

[2] Cirillo, P. and J. Hüsler (2011). Extreme shock models: An alternative perspective. Stat. Probab. Lett. 81(1), 25-30.

[3] Eryilmaz, S. (2012). Generalized $\delta$-shock model via runs. Stat. Probab. Lett. 82(2), 326-331.

[4] Eryilmaz, S. (2013). On the lifetime behavior of a discrete time shock model. J. Comput. Appl. Math. 237(1), 384-388.

[5] Eryilmaz, S. (2017a). Computing optimal replacement time and mean residual life in reliability shock models. Comput. Ind. Eng. 103, 40-45.

[6] Eryilmaz, S. (2017b). On compound sums under dependence. Insurance Math. Econom. 72, 228-234.

[7] Gut, A. (1990). Cumulative shock models. Adv. in Appl. Probab. 22(2), 504-507.

[8] Gut, A. (2001). Mixed shock models. Bernoulli. 7(3), 541-555.

[9] Gut, A. and J. Hüsler (1999). Extreme shock models. Extremes 2(3), 293-305.

[10] Li, Z., Chan L. Y. and Z. Yuan (1999). Failure time distribution under a $\delta$-shock model and its application to economic design of system. Int. J. Rel. Qual. Saf. Eng. 6(3), 237-247.

[11] Li, Z. and X. Kong (2007). Life behavior of $\delta$-shock model. Stat. Probab. Lett. 77(6), 577-587.

[12] Mallor, F. and E. Omey (2001). Shocks, runs and random sums. J. Appl. Probab. 38(2), 438-448.

[13] Neuts, M. F. (1981). Matrix-Geometric Solutions in Stochastic Models: An Algorithmic Approach. The Johns Hopkins University Press, Baltimore MD.

[14] Nikoloulopoulos, A. K. and D. Karlis (2008). Fitting copulas to bivariate earthquake data: The seismic gap hypothesis revisited. Environmetrics 19(3), 251-269.

[15] Ozkut, M. and I. Bayramloglu (Bairamov) (2014). On Marshall-Olkin type distribution with effect of shock magnitude. J. Comput. Appl. Math. 271, 150-162.

[16] Parvardeh, A. and N. Balakrishnan (2015). On mixed $\delta$-shock models. Stat. Probab. Lett. 102, 51-60.

[17] Sumita, U. and J. G. Shanthikumar (1985). A class of correlated cumulative shock models. Adv. in Appl. Probab. 17(2), 347366.

[18] Tank, F. and S. Eryilmaz (2015). The distributions of sum, minima and maxima of generalized geometric random variables. Statist. Papers. 56(4), 1191-1203. 